\title{
Evaluation of Robustness in Time Synchronization for Sensor Networks
}

\author{
Akira Mutazono \\ Graduate School of \\ Information Science and \\ Technology, Osaka University \\ 1-5 Yamadaoka, Suita-shi, \\ Osaka, 565-0871, Japan \\ a-mutazono@ist.osaka- \\ u.ac.jp
}

\author{
Masashi Sugano \\ School of Comprehensive \\ Rehabilitation, Osaka \\ Prefecture University \\ 3-7-30 Habikino, Habikino-shi, \\ Osaka, 583-8555, Japan \\ sugano@rehab.osakafu- \\ u.ac.jp
}

\author{
Masayuki Murata \\ Graduate School of \\ Information Science and \\ Technology, Osaka University \\ 1-5 Yamadaoka, Suita-shi, \\ Osaka, 565-0871, Japan \\ murata@ist.osaka-u.ac.jp
}

\begin{abstract}
In sensor networks, time synchronization between sensor nodes is important because it affects not only the efficiency of information gathering but also energy consumption. Since sensor network environments and sizes change at varying rates, different time synchronization techniques are required for different networks. The pulse-coupled oscillator (PCO) model can be used to achieve local interactions between individuals for the synchronization of entire networks. Other methods also exist, such as the multi-hop reference broadcast synchronization (RBS) method which synchronizes the entire network by transmitting the differences in the timers of reference nodes through networks divided into clusters. We compare here the influence of jitter delay and packet loss resulting from the lower layer protocol on these two techniques. We also investigate the influence on the performance of both methods on network size and wireless channel quality. The simulation results show the conditions in which each method performs better.
\end{abstract}

\section{Keywords}

sensor networks, synchronization, pulse coupled oscillator, CSMA/CA, multi-hop RBS

\section{INTRODUCTION}

Continued improvements in wireless technology that provides low-cost, compact and reliable sensor devices are focusing research on sensor nodes networks from which these devices are comprised [1]. In a sensor network, the time synchronization between sensor nodes is becoming an important feature. For example, energy consumption is a crucial problem for sensor networks, as each sensor node has only the limited power resources of a low capacity battery. Sleep control is one efficient power-saving technique, which when

Permission to make digital or hard copies of all or part of this work for personal or classroom use is granted without fee provided that copies are not made or distributed for profit or commercial advantage and that copies bear this notice and the full citation on the first page. To copy otherwise, to republish, to post on servers or to redistribute to lists, requires prior specific permission and/or a fee.

Bionetics'07 December 10-13, 2007, Budapest, Hungary.

Copyright 2007 ICST 978-963-9799-11-0. used, ensures a sensor node wakes up only when it is required to work [2]. In addition, data gathering becomes more efficient when the sensor nodes transmit information at coordinated times [3]. These power-saving techniques cannot be implemented without the synchronization of all sensor nodes.

Reference Broadcast Synchronization (RBS) [4] has been proposed as the time synchronization technique for use with a wireless network. This technique realizes an exact synchronization by not absolute time but relative time. The advantage of RBS is that it is not affected by send time or access time, which occurs in the MAC layer, because it uses relative time. However, as RBS uses centralized control, it is inapplicable if the network size exceeds transmission range. Therefore, multi-hop RBS is being applied to large-scale networks [4]. However, multi-hop RBS which is centralized time synchronization control can achive a accuracy synchronization, there are some problems in realstic sensor networks (i.e., scalability and packet loss).

Increasing importance is being placed on distributed control as a way to combat these problems. In particular, attention is being paid to the control method which is inspired by the synchronization phenomena seen in a nature. Some papers have defined the efficiency of bio-inspired approach [5]. Then we are motivated to find out the superiority of bio-inspired method against centralized method also in time synchronization control. The pulse-coupled oscillator (PCO) is modeled on biological systems, such as group of fireflies or cardiac pacemaker cells [6]. Past research has evaluated the performance characteristics of the PCO, but has rarely compared it with other synchronization control techniques $[7,8]$. Those studies that do evaluate PCO, do so only under ideal conditions and do not consider the influences of, for example, delays and packet losses that have a marked effect on precision in synchronization and time to synchronization. Moreover, although the goal of those papers is rapid time synchronization based on the communication of pulses in spite of usual packets, it is not realistic from an economic viewpoint to prepare a circuit for this exclusive use. In this paper, we apply packet level PCO that works as overlay of the packet transmission based on IEEE 802.15.4 for applications that do not need high accuracy and high-speed synchronization. We also consider the effects of the low layers in the comparative evaluations of PCO and multi-hop RBS. Carrier Sense Multiple Access with Collision 
Avoidance (CSMA/CA), which is used in IEEE 802.15.4 as standard protocol in sensor networks, works as the transmission protocol on the MAC layer. We implemented PCO and multi-hop RBS as synchronization mechanisms in the high layer above CSMA/CA, compared PCO and multi-hop RBS using simulations, and investigated the network environment for which each method is most suited.

The organization of this paper is follows. We first explain the network models and define synchronization in Section 2. In Section 3, we describe synchronization with PCO. And in Section 4, we show how multi-hop RBS operates. In Section 5 , we present the results of our simulations and conclude by discussing plans for future work.

\section{SYSTEM DEFINITION}

\subsection{Network Model}

All the sensor nodes have the same capability, and have an oscillator indicating internal time. It is assumed that the cycle of all the oscillators is the same. Internal time of oscillator $C_{i}(t)$ is expressed as Eq. (1) using clock drift $a_{i}(t)$, offset $b_{i}(t)$, and clock cycle $T_{i}$ of node $i$. In this paper, we assume that none of sensor nodes is synchronized at the initial state, and the offset is set at random.

$$
C_{i}(t)=a_{i}(t) T_{i}+b_{i}(t)
$$

Sensor nodes use CSMA/CA as their MAC layer protocol. With CSMA/CA, carrier sensing is done before the packet is transmitted, and it is known whether a packet can access the channel. We adopt the CSMA/CA protocol according to IEEE 802.15.4.

\subsection{Synchronization Model}

In real networks, it is difficult for all sensor nodes to be completely synchronized due to transmission delay, interface, and packet loss. In addition, the required precision of the synchronization differs according to the application. Therefore, we use a synchronization window $W$ as the parameter to determine a synchronization and use this as an index of target synchronous accuracy [9]. Consider the relative offset $z_{i j} \in[-0.5,0.5]$ between node $n_{i}$ and $n_{j}$, which has the internal time $b_{i}, b_{j} \in[0,1]$. $z_{i j}$ is expressed as

$$
z_{i j}=\left(\left(b_{j}-b_{i}+1.5\right) \bmod 1.0\right)-0.5
$$

We define the synchronization group $G_{i}(w)$ which starts from node $n_{i}$ and synchronization window size $w$ which decides the size of group. Then we find the biggest group.

$$
\begin{gathered}
G_{i}(w)=\left\{n_{j} \mid 0<z_{i j}<w\right\} \\
S_{i}=\left|G_{i}(w)\right| \\
S_{k}=\max _{\forall i} S_{i}
\end{gathered}
$$

where $S_{i}$ is the number of nodes in group $G_{i}(w)$ and $S_{k}$ is the ratio of synchronized nodes. If $S_{k}$ is equivalent to the number of all sensor nodes, the group $G_{k}$ is in the complete synchronous state.

\section{BIO-INSPIRED TIME SYNCHRONIZA- TION CONTROL}

The distributed communication strategy that we propose is based on packet instead of pulse. This condition makes synchronization difficult, but it is necessary for synchronization control to synchronize real network.

\subsection{Mirollo and Strogatz Model}

The M\&S model [6] is the time synchronization mechanism applied by PCO to sensor network. An oscillator works based on its phase, $\phi \in[0,1]$, representing the internal time and the state, $x \in[0,1]$, representing the phase. Let us consider the set $O=\left\{O_{1}, \cdots O_{N}\right\}$ of $N$ oscillators. Each oscillator has phase $\phi_{i}$ and state $x_{i}$, which changes over time, and $x_{i}$ is given by the function $f_{i}$.

$$
x_{i}=f_{i}\left(\phi_{i}\right)
$$

In particular, $f_{i}(0)=0, f_{i}(1)=1$, and phase $\phi_{i}$ changes 0 to 1 every clock cycle $T_{i}$ and $\frac{d \phi_{i}}{d t}=\frac{1}{T_{i}}$. When a phase reaches 1 , the oscillator fires and the phase is reset to 0 . If oscillator $\tau_{i}$ receives a pulse, then its oscillator increases $\varepsilon$ to its own state, the two oscillators, $O_{i}$ and $O_{j}$, are coupled.

$$
x_{j}\left(\tau_{i}^{+}\right)= \begin{cases}x_{j}\left(\tau_{i}\right)+\varepsilon, & \text { if } x_{j}\left(\tau_{i}\right)+\varepsilon<1 \\ 0, & \text { otherwise }\end{cases}
$$

By giving such a stimulus to each other, a coupled oscillator becomes synchronized over time.

\subsection{Effect of Delay on Time Synchronization}

The M\&S model considers that the effect of the firing of a neighboring node instantaneously takes place, regardless of delay. However, since delay will in fact occur by the time a sensor node fires and transmits information, it is necessary for a node to take account of the delay. In the Reach-back Firefly Algorithm (RFA) [9], the timestamp is used to announce the access delay on MAC layer. First, the sensor node stores the time of firing and transmits data after a waiting period when it is confirmed by CSMA/CA that the channel is free. Thus, the receiving node can know the delay after a source node fires by marking data packet using delay as the time stamp. Although access delay can be indicated with a time stamp, it is not sufficient for the M\&S model to remove the effects of delay, since a phase cannot be changed simultaneously with firing. Additionally, when a phase is changed at the same time as the effect of the firing is experienced, some problems arise. To avoid these problems, even if a sensor node receives information about a firing, the amount of the change in the phase should be stacked at once without being changed. Then, the value of the stack is announced and, the phase is changed after self firing and waiting fixed time waiting time $W . W$ should be longer than the back-off time of CSMA/CA.

\section{CENTRALIZED TIME SYNCHRONIZA- TION CONTROL}

\subsection{Reference Broadcast Synchronization}

Reference broadcast synchronization (RBS) [4] is a time synchronization mechanism that does not use a time stamp. RBS does not set the time of the transmission node and the receiving node but sets the times of nodes receiving from each other. The main benefit of RBS is that it achieves a high accuracy in synchronization which is not affected by access delay. Since the error in the reference time between the nodes is an error until a packet is transmitted and re- 
ceived. The access delay, which is the time until a packet is transmitted, can be eliminated.

Step1. A base node broadcasts a reference packet

Step2. $n$ sensor nodes, which receives the reference packet, store their own reception time $T$.

Step3. A reception node exchanges reception times with other sensor nodes that receive the reference packet.

Step4. A reception node calculates the average time of error offset $[i]$ of the exchanged reception time and its own reception time.

where $n$ is the number of the sensor nodes within the transmission range of base node, and $T_{r}$ is the time when sensor node $r$ receives the reference packet. The offset $[i]$ is given by Equation (8).

$$
\operatorname{offset}[i]=\frac{1}{n} \sum_{k=1}^{n}\left(T_{i}-T_{k}\right) \quad \forall i \in n
$$

\subsection{Multi-hop RBS}

RBS can be effective only when all sensor nodes are arranged within the communication range of a base node. In other words, RBS can not synchronize large scale networks because of the communication distances elimination of the base nodes. Therefore, the whole network is divided into clusters and RBS is applied to every cluster. A sensor node called a gateway node, which belongs to two or more clusters, translates the relative time between clusters. The mechanism for transmitting synchronous time information to the whole network is called multi-hop RBS.

In multi-hop RBS, the sensor node called the sink plays a key role in controlling the synchronization of the whole network. First, the sink transmits the schedule for executing RBS to a cluster head by multi-hop communication. The cluster head that receives the schedule information carries out RBS within its own cluster, and when it is confirmed that all the sensor nodes in the cluster can be synchronized, it tells that RBS was completed. After a sink confirms that RBS has been carried out by all the clusters, it broadcasts the synchronization time information in its own cluster to set the time of the entire network at the synchronous time of its own cluster. The gateway node that receives synchronous time information spreads it to the next cluster while it calculates the error in the synchronous time between clusters and notifies other sensor nodes in a cluster of it. In this way, time synchronous information spreads through out the network, and all sensor nodes are synchronized.

\section{SIMULATION RESULTS}

In this paper, two versions of the simulation program that operate at higher layer above CSMA/CA were formulated, one with PCO and one with multi-hop RBS. The performance of these two versions will now be evaluated and compared. The observation area in which sensor nodes are deployed is circular. The parameters that were used in the simulations are shown in Table 1 . We use various metrics to evaluate the simulation results: the ratio of synchronized nodes, the probability of synchronization, the time to synchronization, and the clock variance.

In the PCO program, contrary stimuli can be given mutually and a completely synchronous state can collapse because the network that reached the synchronous state was unstable. Therefore, we consider a network synchronized when
Table 1: Default parameter settings

\begin{tabular}{|c|c|}
\hline parameter & value \\
\hline \hline Number of sensor nodes & 200 \\
\hline Radius of monitoring region & $100 \mathrm{~m}$ \\
\hline Communication range & $50 \mathrm{~m}$ \\
\hline Packet loss rate & 0.001 \\
\hline Synchronous window size & 0.1 \\
\hline Stimulation of fire $\varepsilon(\mathrm{PCO})$ & 0.0008 \\
\hline Firing cycle $T$ (PCO) & $0.16 \mathrm{sec}$ \\
\hline Maximum simulation time $(\mathrm{PCO})$ & $100 \mathrm{sec}$ \\
\hline
\end{tabular}

PCO stays for five continuous cycles in a completely synchronous state. We consider a network as synchronized when multi-hop RBS cluster synchronous information spreads to all the clusters and the network is in a completely synchronous state. The time taken is assumed to be the time to synchronization. Similarly, the time synchronous accuracy is measured by the variance of the phase of the sensor node.

$$
\begin{gathered}
v=\frac{1}{n} \sum_{i=1}^{n} e_{i}^{2} \\
e_{i}= \begin{cases}\bar{x}-x_{i}, & \left|\bar{x}-x_{i}\right|<0.5 \\
1-\left|\bar{x}-x_{i}\right|, & \text { otherwise }\end{cases}
\end{gathered}
$$

where $n$ is the number of nodes, $x_{i}$ is the phase of sensor node $i, \bar{x}$ is the phase average of the largest group, and $e_{i}$ is the error between $x_{i}$ and $\bar{x}$. Reliability of data was verified by using confidence interval of $95 \%$ with 400 trials.

\subsection{Network Scalability}

In sensor networks, lots of devices can be deployed over a wide area, so it is important for synchronous technique to be able to synchronize networks of various size. Figure 1 shows how the size of the network affects each synchronization mechanism. Figure 1(b), 1(c) shows that multi-hop RBS establishes precise synchronization in a short time in high node density environments. However, multi-hop RBS loses a significant number of synchronized nodes as the density of sensor nodes decreases (Figure 1(a)). By contrast, PCO shows stable performance. This is because of the lack of connectivity of multi-hop RBS compared with PCO. It is necessary for RBS to communicate between the sensor nodes on different edges of a cluster, and the radius of a cluster is limited to half of transmission range. Thus, PCO and multi-hop RBS show relative performance advantages over each other based on network environment, and which technique is used should be decided based on the application and node density in which it will be used.

\subsection{Robustness to Packet Loss}

Synchronous techniques must be robust to packet loss because the radio technology used in a sensor network is fragile and the packets do not always reach the destination. Figure 2 shows the effects of packet loss on the two synchronous methods. Almost all sensor nodes can be synchronized by both synchronous techniques even in unstable environments where packet loss occurs frequently. This is because PCO compensates for the effects of packet loss by causing sensor nodes to give repeated mutual stimulation, and multi-hop 


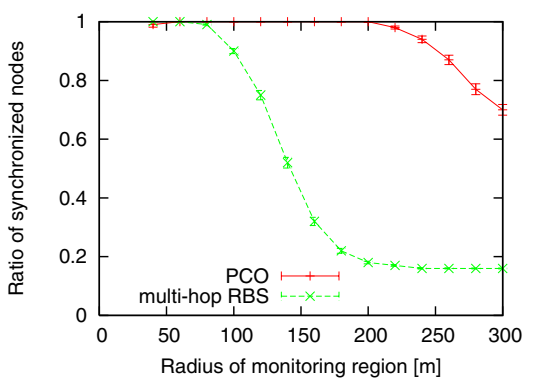

(a) PCO can synchronize all sensor nodes in a much wider monitoring region than multi-hop RBS

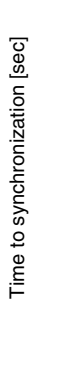

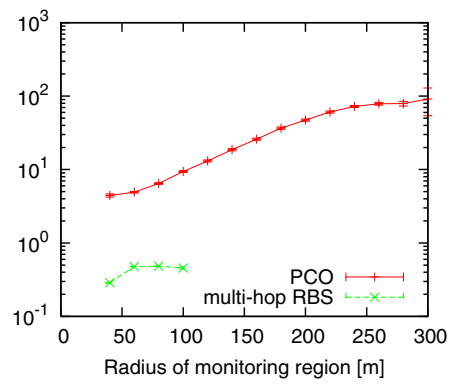

(b) Multi-hop RBS is not able to synchronize a network where the radius of monitoring region is larger than $110 \mathrm{~m}$

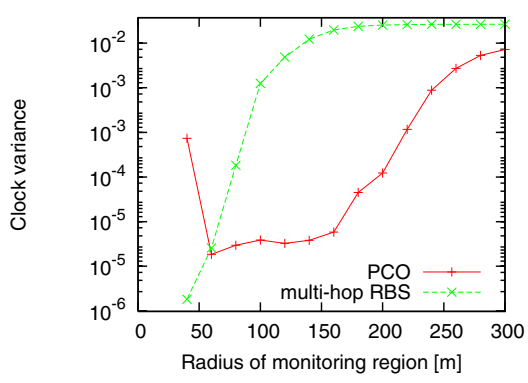

(c) Multi-hop RBS can accurately synchronize a network in a small monitoring region

Figure 1: Evaluation over the size of the monitoring region

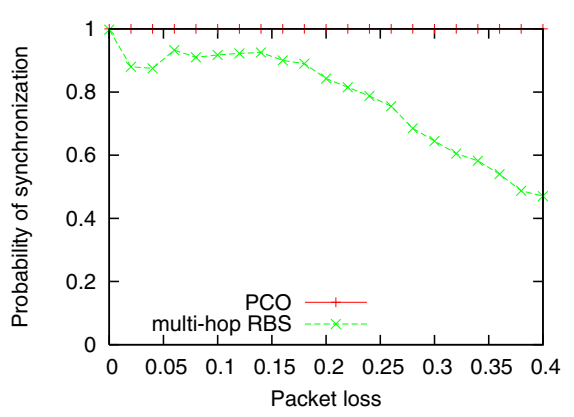

Figure 2: Packet loss performance. Only few sensor nodes are out of synchronization in multi-hop RBS due to expanding of the error caused by the packet loss

RBS retransmits the packet if the ACK packet is not returned. However, in multi-hop RBS, almost all sensor nodes can be synchronized, but it is difficult for all sensor nodes to carry out a complete time synchronization since the synchronous error produced by the packet loss within the cluster increases by a synchronous time spreading between clusters by multi-hop communications. For these reasons, it is found that PCO is effective in an unstable communication environment and complete time synchronization is needed.

\section{CONCLUSION}

We comparatively evaluated two time synchronization techniques, PCO and multi-hop RBS, from the viewpoint of scalability and robustness to packet loss in consideration of delay by CSMA/CA. We found that, bio-inspired time synchronization control with PCO, can achieve very stable time synchronization regardless of radio quality over a wide observation area. We also found that multi-hop RBS, centralized time synchronization control, can establish highly precise time synchronization in a short time at high node densities, and in high radio quality environments. We leave to future work the discussion of the energy efficiency of bioinspired synchronization method.

\section{ACKNOWLEDGMENTS}

This research was supported in part by "Special Coordination Funds for Promoting Science and Technology: Yuragi Project" and Grant-in-Aid for Scientific Research (contract number (C) 19500060) of the Ministry of Education, Culture, Sports, Science and Technology.

\section{REFERENCES}

[1] I. Akyildiz, W. Su, Y. Sankarasubramaniam and E. Cayirci. Wireless sensor networks: A survey. Computer Networks, 38:393-422, 2002.

[2] F. Sivrikaya and B. Yener. Time synchronization in sensor networks: A survey. IEEE Network Magazine, 18(4):45-50, 2004.

[3] N. Wakamiya and M. Murata. Synchronization-based data gathering scheme for sensor networks. IEICE Trans. on Commun., E88-B:873-881, 2005.

[4] J. Elson, L. Girod and D. Estrin. Fine-grained network time synchronization using reference broadcasts. In Proceedings of Operational Systems and Design Implementation (OSDI), pages 147-163, 2002.

[5] S. Barbarossa and G. Scutari. Bio-Inspired Sensor Network Design. IEEE Signal Processing Magazine, 24:26-35, 2007.

[6] R. E. Mirollo and S. H. Strogatz. Synchronization of pulse-coupled biological oscillators. Journal on Applied Mathematics, 50(6):1645-1662, 1990.

[7] Y. W. Hong and A. Scaglione. A scalable synchronization protocol for large scale sensor networks and its applications. IEEE Journal on Selected Areas in Commun., 23(5):1085-1099, 2005.

[8] S. F. Bush. Low-energy sensor network time synchronization as an emergent property. In Proceedings of the 14th ICCCN, pages 93-98, 2005.

[9] G. Werner-Allen, G. Tewari, A. Patel, M. Welsh and R. Nagpal. Firefly-inspired sensor network synchronicity with realistic radio effects. In Proceedings of the 3rd ACM SenSys '05, pages 142-153, 2005. 